\title{
Kennedy Disease Misdiagnosed as Polyneuropathy, Organomegaly, Endocrinopathy, Monoclonal Gammopathy, and Skin Changes (POEMS) Syndrome: A Case Report
}

\author{
Min Yuan ${ }^{a}$ Weiping Chen ${ }^{a}$ Huangyan Zhou $^{b}$ Zhilong Xiao ${ }^{a}$ Wei Wang $^{\mathrm{a}}$

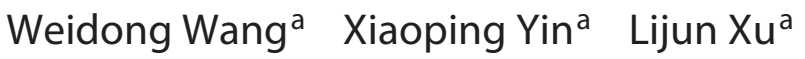 \\ a Department of Neurology, The Second Affiliated Hospital of Nanchang University, and ${ }^{\mathrm{b}}$ Department of Immunology \\ and Pathogenic Biology, The Basic Medical College of Nanchang University, Nanchang, China
}

\section{Key Words}

Kennedy disease · POEMS syndrome · Gynecomastia · Gene analysis

\begin{abstract}
Objective: The aim of this paper was to report the first case of Kennedy disease misdiagnosed as polyneuropathy, organomegaly, endocrinopathy, monoclonal gammopathy, and skin changes (POEMS) syndrome. Clinical Presentation and Intervention: A 58-year-old Chinese man presented with limb numbness, progressive limb proximal weakness, lymph node and thyroid enlargement, edema, pigmentation in the lower limb, and obvious gynecomastia, which was initially diagnosed as POEMS syndrome and was treated with dexamethasone and small doses of cyclophosphamide without any improvement after 6 months. Finally, the patient diagnosis was confirmed as Kennedy disease (KD) by gene analysis. Conclusion: This case suggests that clinicians should pay more attention to the differential diagnosis between KD and POEMS syndrome. Gene analysis was helpful in detecting this rare confusing disease in this patient.
\end{abstract}

(c) 2015 S. Karger AG, Basel

\begin{tabular}{ll}
\hline KARGER & $\begin{array}{l}\text { ( 2015 S. Karger AG, Basel } \\
1011-7571 / 15 / 0253-0286 \$ 39.50 / 0 \quad \text { Karger }\end{array}$ \\
E-Mail karger@karger.com & \\
www.karger.com/mpp & $\begin{array}{l}\text { This article is licensed under the Creative Commons Attribution- } \\
\text { NonCommercial-NoDerivatives 4.0 International License (CC BY- } \\
\text { NC-ND) (http://www.karger.com/Services/OpenAccessLicense). } \\
\text { Usage and distribution for commercial purposes as well as any dis- } \\
\text { tribution of modified material requires written permission }\end{array}$
\end{tabular}

\section{Introduction}

Kennedy disease (KD), also known as X-linked spinal bulbar muscular atrophy, is a lower motor neuron degenerative disease with onset in adulthood and is characterized by slowly progressive proximal weakness, medullary involvement, muscle atrophy, and widespread muscle bundle fibrillation accompanied by postural tremor and nonneurological symptoms, such as gynecomastia $[1,2]$. Gene analysis can provide a diagnostic basis for this disease. Polyneuropathy, organomegaly, endocrinopathy, monoclonal gammopathy, and skin changes (POEMS) syndrome is a rare paraneoplastic syndrome caused by an underlying plasma cell disorder [3]. The disorder may have overlapping clinical manifestations with $\mathrm{KD}$. It is necessary for clinicians to have a high index of suspicion to distinguish them. Here we present a case of KD initially misdiagnosed as atypical POEMS syndrome. 


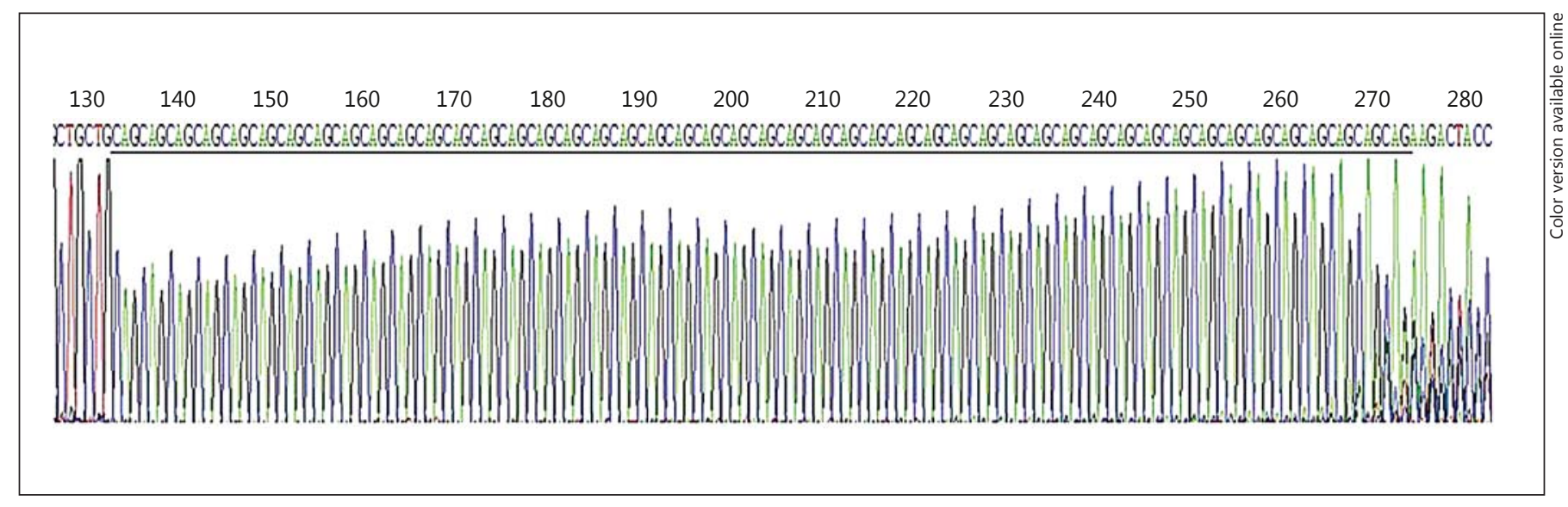

Fig. 1. Gene analysis result for the patient. The horizontal line shows the CAG repeat is 47.

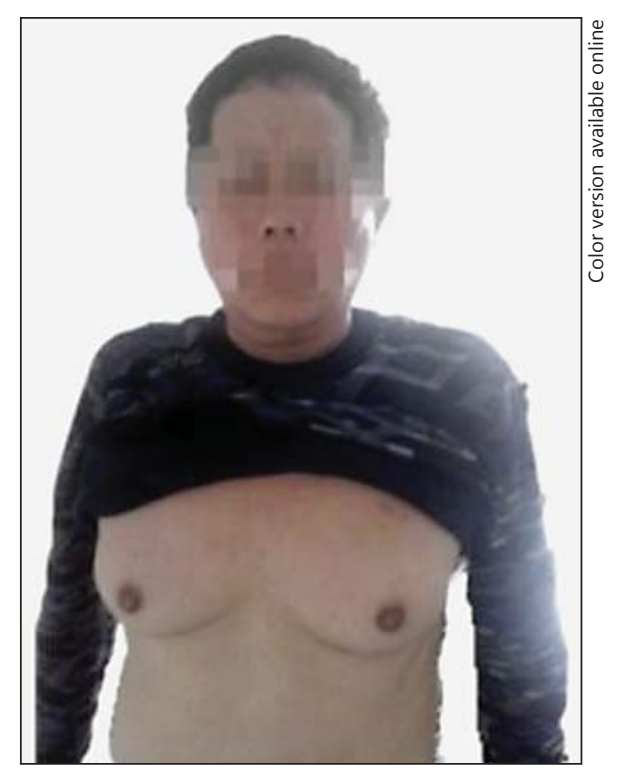

Fig. 2. The male patient with obvious gynecomastia.

\section{Case Report}

Written informed consent was obtained from the patient and the patient's son for publication of this case report and accompanying images The above Chinese man, who developed muscular weakness more than 10 years previously, first noticed difficulty with ambulating, especially climbing stairs and standing up from a seated position, and subsequently had dual arm fatigue accompanied with double lower limb numbness. At that time the patient did not pay any attention. The illness persisted and developed gradually. He started to experience aggravation of lower extremity weakness 4 years ago, with limb numbness, and he also complained of some difficulty with swallowing liquids, as well as decreasing libido and a significant lack of any sexual desire. Physical examination showed that he had two nontender enlarged lymph nodes, about $0.5 \times 0.5 \mathrm{~cm}$ in his middle neck triangles, and mild enlargement in the thyroid gland. Compared with the right side of the limb, the left lower limb skin color was darker. X-ray also showed the presence of diffuse osteopenia. The M-protein was not detected in his blood or urine but the serum $\gamma$-globulin ratio was significantly higher. These characteristics were consistent with the diagnostic criteria of POEMS syndrome. He was initially diagnosed with atypical POEMS syndrome upon consultation with a hematologist, and received dexamethasone and small doses of cyclophosphamide for 6 months without any improvement. When we met the patient again 3 months ago, the above symptoms had gotten worse, with walking instability, turning difficulty, and lower extremity edema.

Neurological examination revealed blurred articulation, dualside muscle tiredness, and incomplete eyelid closure on the right side. In addition, the nasolabial fold was lighter on the right than on the left side. He could not cheer and whistle (with right-sided uvula), his gag reflex disappeared, he once had myokymia in the chin muscles, and he sometimes experienced mild tongue atrophy with fasciculations. The patient had obvious gynecomastia. Strength testing demonstrated progressive proximal weakness in the upper and lower extremities. Laboratory examinations showed increased creatine kinase (765.8 IU/l), estradiol (50.48 $\mathrm{pg} / \mathrm{ml})$, and prolactin $(28.39 \mathrm{ng} / \mathrm{ml})$. Serum protein electrophoresis indicated the following: albumin 59.0, $\alpha_{1}$-globulin 3.5, $\alpha_{2^{-}}$ globulin 7.6, $\beta$-globulin 7.0, and $\gamma$-globulin 23.0. Urine and serum protein electrophoresis still showed no evidence of monoclonal antibodies. A lumbar MRI scan also showed enlargement of the retroperitoneal lymph node. Peripheral nerve conduction velocity in the limbs prompted attenuation of tibial nerve compound action potential amplitude; motor nerve conduction velocity was normal. The sensory potential amplitudes of the median and ulnar nerves were significantly attenuated. Superficial sural and pe- 
roneal nerve sensory potentials did not elicit positive records. F waves obtained from stimulation of the median nerve appeared lower than 50\%. Electromyography (EMG) showed a large number of normal-phase fibrillation potentials when the muscles relaxed; with mild muscle contraction, the reaction time of motor units extended, and the amplitude increased, and when the muscles vigorously contracted, the number of motor units decreased, rendering it as a simple phase. In conclusion, EMG showed widespread neuropathic damage. The results of gene analysis also suggest that the androgen receptor gene in exon 1 of the CAG trinucleotide repeat number is 47 , confirming the suspected diagnosis of KD (fig. 1, 2).

\section{Discussion}

This case highlights the difficulties involved in the differential diagnosis of $\mathrm{KD}$ from $\mathrm{POEMS}$ syndrome through the patient's clinical symptoms and physical examination. Gene analysis is essential for the diagnosis of KD due to the high rate of misdiagnosis in practical clinical work, as has been previously reported $[4,5]$. POEMS syndrome is a rare paraneoplastic syndrome associated with plasma cell dyscrasia that has overlapping clinical manifestations with $\mathrm{KD}$. At first, we were confident that the above case fulfilled the diagnosis of atypical POEMS syndrome, so a bone marrow biopsy was not performed after the onset of his symptoms. Although the M-protein was not detected in the blood and urine of the patient, we found the serum $\gamma$-globulin ratio of the patient was significantly higher, and studies have shown that the incidence of M-proteins in patients with POEMS syndrome is not $100 \%$. We know now, however, that the initial diagnosis was not appropriate, and we hope that clinicians will pay more attention and thus prevent the occurrence of such phenomena in the future.

$\mathrm{KD}$ is an $\mathrm{X}$-linked recessive disorder due to a dynamic mutation in the androgen receptor gene [2]. Although KD has overlapping clinical manifestations with POEMS syndrome, there are distinguishing characteristics. The major mandatory criteria of $\mathrm{KD}$ include progressive proximal weakness, medullary involvement, asymmetrical muscle weakness, muscle atrophy, gynecomastia, and sexual dysfunction $[1,2]$. This is different from POEMS syndrome that mainly involves symptoms of polyneuropathy and monoclonal plasma proliferative disorders [3]. Furthermore, the EMG in KD often shows widespread neuropathic damage. Muscle fibrillation potentials appear, with positive sharp waves in the resting state. Motor nerve conduction velocity is normal, but the sensory nerve action potential amplitude often decreases, as does the sensory nerve conduction velocity. The muscle biop- sy usually shows neurogenic damage and occasionally muscle damage $[1,6]$.

Studies have found that the pathogenesis of $\mathrm{KD}$ is androgen receptor mutations, which are caused by an expansion of the CAG trinucleotide repeat in the androgen receptor gene on chromosome Xq11-12 [6], making androgen receptor protein in polyglutamine regional augmentation [7]. Its toxicity and abnormal degradation eventually cause cell dysfunction and transgender deaths $[1,8]$. Most of the patients are male, and the gold standard for diagnosis is gene analysis. In healthy people, the CAG repeat range of gene analysis is from 17 to 26; the new European Neuroscience Association (EFNS) guidelines show that patients can be diagnosed with Kennedy disease if the CAG repetitive sequence number $\geq 35$ [9]. In this study, the patient's CAG repetitive sequence number is 47 , which is consisted with the diagnostic criteria of KD. However, the patient has a son who has no similar symptoms, and whose CAG repetitive sequence number is 27 , which may indicate that $\mathrm{KD}$ is a rare $\mathrm{X}$-linked recessive disorder.

As we all know, currently, there is no specific treatment for $\mathrm{KD}$ and it is still dominated by symptomatic treatment [6]. Studies have shown an inhibition of abnormal protein accumulation, and applications with androgen deprivation are the basic treatment strategies for polyglutamine disease. As $\mathrm{KD}$ is androgen dependent, one study has suggested that blocking androgen action may be an effective treatment [10], but the effects have not been clearly proven in clinical trials [7]. Therefore, the specific pathogenesis and treatment methods of KD are worthy of further study and need more research to confirm.

\section{Conclusion}

This was a case of initial misdiagnosis: of POEMS syndrome instead of KD. This suggests that clinicians should pay more attention to the differential diagnosis of KD and POEMS syndrome and that gene analysis is essential to differentiate these rare confusing diseases. 


\section{References}

$>1$ Kennedy WR, Alter M, Sung JH: Progressive proximal spinal and bulbar muscular atrophy of late onset. A sex-linked recessive trait. Neurology 1968;18:671-680.

$>2$ Sperfeld AD, Karitzky J, Brummer D, et al: Xlinked bulbospinal neuronopathy: Kennedy disease. Arch Neurol 2002;59:1921-1926.

$>3$ Chu BF, Shana'Ah A, Hofmeister CC, et al: Long-term therapy with lenalidomide in a patient with POEMS syndrome. Eur J Case Rep Intern Med 2014;1:000093.

4 Harutunian GM, Beydoun SR, Rison RA: Kennedy disease misdiagnosed as polymyo- sitis: a case report. BMC Res Notes 2013;6: 389.

5 Fu MH, Lan MY, Liu JS, et al: Kennedy disease mimics amyotrophic lateral sclerosis: a case report. Acta Neurol Taiwan 2008;17:99-103.

6 Finsterer J: Perspectives of Kennedy's disease. J Neurol Sci 2010;298:1-10.

7 Katsuno M, Watanabe H, Yamamoto M, et al: Potential therapeutic targets in polyglutamine-mediated diseases. Expert Rev Neurother 2014;14:1215-1228.

8 Adachi H, Waza M, Tokui K, et al: CHIP overexpression reduces mutant androgen recep- tor protein and ameliorates phenotypes of the spinal and bulbar muscular atrophy transgenic mouse model. J Neurosci 2007;27:51155126.

9 Burgunder JM, Schols L, Baets J, et al: EFNS guidelines for the molecular diagnosis of neurogenetic disorders: motoneuron, peripheral nerve and muscle disorders. Eur J Neurol 2011;18:207-217.

10 Jordan CL, Lieberman AP: Spinal and bulbar muscular atrophy: a motoneuron or muscle disease? Curr Opin Pharmacol 2008;8:752758 